\title{
Identifying the Real Minority Carrier Lifetime in Nonideal Semiconductors: A Case Study of Kesterite Materials
}

\author{
Charles J. Hages,* Alex Redinger, Sergiu Levcenko, Hannes Hempel, Mark J. Koeper, \\ Rakesh Agrawal, Dieter Greiner, Christian A. Kaufmann, and Thomas Unold*:
}

Time-resolved photoluminescence (TRPL) is a powerful characterization technique to study carrier dynamics and quantify absorber quality in semiconductors. The minority carrier lifetime, which is critically important for high-performance solar cells, is often derived from TRPL analysis. However, here it is shown that various nonideal absorber properties can dominate the TRPL signal making reliable extraction of the minority carrier lifetime not possible. Through high-resolution intensity-, temperature-, voltage-dependent, and spectrally resolved TRPL measurements on absorbers and devices it is shown that photoluminescence (PL) decay times for kesterite materials are dominated by minority carrier detrapping. Therefore, PL decay times do not correspond to the minority carrier lifetime for these materials. The lifetimes measured here are on the order of hundreds of picoseconds in contrast to the nanosecond lifetimes suggested by the decay curves. These results are supported with additional measurements, device simulation, and comparison with recombination limited PL decays measured on $\mathrm{Cu}(\mathrm{In}, \mathrm{Ga}) \mathrm{Se}_{2}$. The kesterite material system is used as a case study to demonstrate the general analysis of TRPL data in the limit of various measurement conditions and nonideal absorber properties. The data indicate that the current bottleneck for kesterite solar cells is the minority carrier lifetime.

\section{Introduction}

Time-resolved photoluminescence (TRPL) is a useful technique to characterize charge carrier dynamics in semiconducting materials. This technique can be used to extract

Dr. C. J. Hages, Dr. A. Redinger, Dr. S. Levcenko, H. Hempel,

Dr. T. Unold

Department of Structure and Dynamics of Energy Materials

Helmholtz-Zentrum für Materialien und Energie

Hahn-Meitner-Platz 1, 14109 Berlin, Germany

E-mail: charles.hages@helmholtz-berlin.de; unold@helmholtz-berlin.de

M. J. Koeper, Prof. R. Agrawal

Davidson School of Chemical Engineering

Purdue University

480 Stadium Mall Dr., West Lafayette, IN 47901, USA

Dr. D. Greiner, Dr. C. A. Kaufmann

Institute Competence Centre Photovoltaics Berlin

Helmholtz-Zentrum Berlin für Materialien und Energie

Schwarzchildstraße 3, 12489 Berlin, Germany

The ORCID identification number(s) for the author(s) of this article can be found under https://doi.org/10.1002/aenm.201700167.

DOI: 10.1002/aenm.201700167 relevant optoelectronic properties such as the minority carrier lifetime, mobility, and charge carrier density in absorbers and devices. ${ }^{[1-4]}$ For kesterites, TRPL is often used as a metric for absorber quality for comparing and optimizing device performance..$^{[5-25]}$ Characteristic photoluminescence (PL) decay times of a few nanoseconds are commonly reported and associated with the minority carrier lifetime $\tau_{\mathrm{n}}$ for this material. However, little experimental justification is often provided for the conclusion that the reported PL decay times should be related to the minority carrier lifetime of the absorber.

One of the challenges of interpreting TRPL data is that numerous charge carrier dynamics can influence the measured PL decay. Detailed theoretical and experimental discussion regarding the role of various absorber properties and measurement conditions on the PL decay signal are reported by Maiberg et al., $[2,26,27]$ Ahrenkiel, ${ }^{[1,28]}$ and Kuciauskas et al. ${ }^{[4]}$ First, carrier redistribution processes can dominate the early decay signal, as illustrated in Figure 1a. As generation follows a Beer-Lambert absorption profile, carrier diffusion will occur during initial times following the excitation pulse; this process may include energetic relaxation of carriers due to gradients in the absorber or due to potential (i.e., electrostatic or band gap) fluctuations, as illustrated. Following carrier redistribution, radiative and nonradiative recombination in addition to properties such as surface recombination, carrier drift in an electric field, absorber inhomogeneity, material degradation, and minority carrier trapping can have a significant impact in the measured signal. ${ }^{[1,2,27,29-32]}$ An illustration of various process which influence the TRPL decay is shown in Figure 1b. Further details regarding the role of processes shown in Figure 1 in relation to the PL decay are discussed throughout the text. These effects make characterization of nonideal or highly defective materials, such as $\mathrm{Cu}_{2} \mathrm{ZnSnSe}_{4}$ (CZTSe) or $\mathrm{Cu}_{2} \mathrm{ZnSn}(\mathrm{S}, \mathrm{Se})_{4}$ (CZTSSe), difficult. While isolating the impact of various sample properties on the PL decay is possible, as demonstrated in this work, such analysis is often overlooked in the interpretation of PL decay times for kesterites, which can result in incorrect conclusions regarding fundamental device properties. 
(a) Initial Redistribution
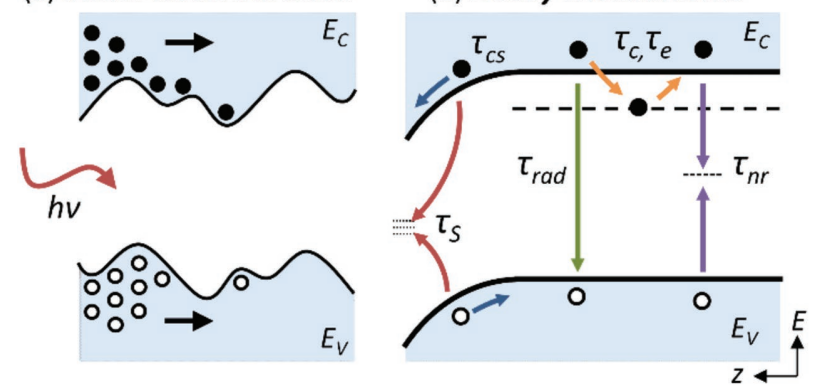

Figure 1. a) Illustration of the initial carrier redistribution following BeerLambert absorption upon excitation. The black arrows indicate axial diffusion of carriers, which may involve energetic relaxation into local potential minima. b) Illustration of various charge-carrier dynamics measured with TRPL. $\tau_{\text {rad }}$ indicates the radiative recombination lifetime, $\tau_{\mathrm{nr}}$ nonradiative recombination lifetime, $\tau_{\mathrm{s}}$ surface recombination lifetime, $\tau_{\mathrm{cs}}$ charge-separation time due to an electric field, and $\tau_{\mathrm{c}}$ and $\tau_{\mathrm{e}}$ minority carrier capture and emission times, respectively, into a trap state(s). Potential fluctuations shown in (a) are not depicted in (b) due to the relatively extended length scale of the illustration.

Major discrepancies are apparent in the reported analysis of TRPL data for kesterites. First, no correlation between the measured PL decay times and device performance over a wide range (1\%-12\%) of device efficiencies (shown in Figure 2 ) and open-circuit voltage $V_{\mathrm{OC}}$ (see the Supporting Information) can be found from published TRPL data. ${ }^{[5-24]}$ These results are in contrast to that found from TRPL analysis of chalcopyrites and CdTe where a correlation with device efficiency and $V_{\mathrm{OC}}$ is

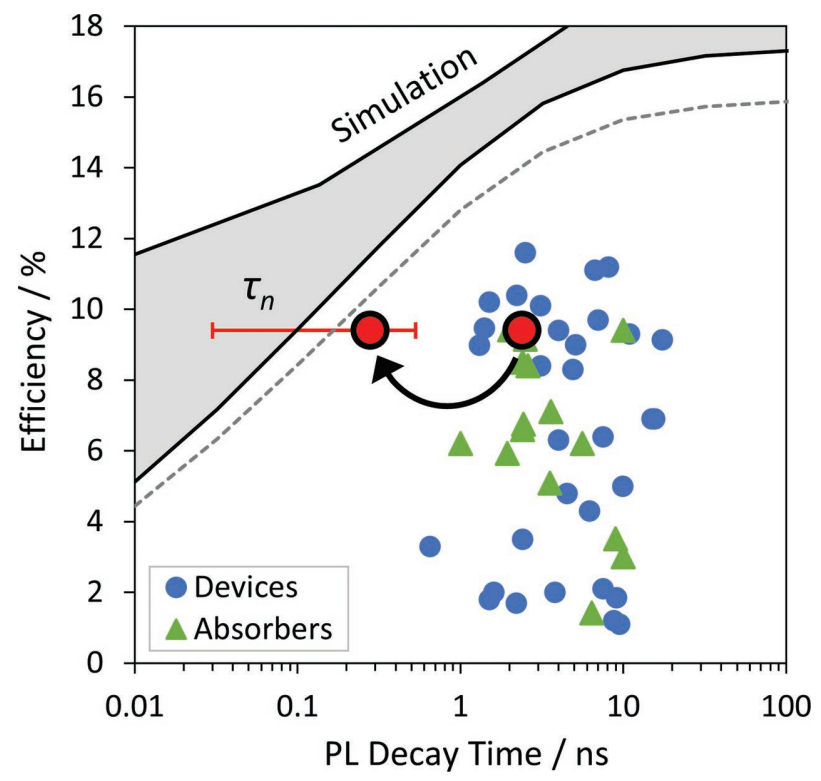

Figure 2. Comparison of PL decay times measured on kesterite absorbers and devices versus their corresponding device power conversion efficiency (PCE); decay times for absorbers are measured prior to device completion or on equivalent absorbers to that of the corresponding device PCE. Data are taken from refs. [5-24]. The solid lines represent device simulation results bounded by $S_{\mathrm{F}}=10^{2}-10^{6} \mathrm{~cm} \mathrm{~s}^{-1}$ and $S_{B}=10^{2}-10^{7} \mathrm{~cm} \mathrm{~s}^{-1}$; the dashed line represents a lower bound on the efficiency for a reduced mobility gap $E_{\mu}=E_{\mathrm{PL}, \text { Peak }}$. The data point labeled $\tau_{\mathrm{n}}$ represents the minority carrier lifetime value estimated in this work, with the corresponding measured PL decay time as indicated. clear, as $\tau_{\mathrm{n}}$ is a measure of recombination losses. ${ }^{[33-36]}$ However, for kesterites the connection between PL decay time and the assumed $\tau_{\mathrm{n}}$ is not apparent, as the reported decay times represent arbitrary measurement excitation conditions and data analysis procedures; characteristic decay times from a variety of fitting regions and techniques are reported for measured TRPL data. Additionally, PL decay times as high as 1-10 ns can be found for devices with $<4 \%$ efficiencies.

Second, TRPL data are commonly reported from measurements on completed kesterite devices, ${ }^{[5-12,16-18,24]}$ despite the significant effect that charge-carrier separation in an electric field has on PL decays. ${ }^{[2,4,30,31,37]}$ A more concerning observation is that no difference between PL decays measured on absorbers and measured on devices is characterized for kesterites, as seen in Figure 2 where measurements on absorbers and devices are distinguished, and also reported for high-efficiency kesterite devices/ absorbers $^{[25]}$ and devices/absorbers reported here (Section 2.2). These results illustrate that a straightforward recombination limited PL decay model is inapplicable for kesterites.

Finally, PL decay times commonly reported for kesterites do not match the corresponding device performance or $V_{\mathrm{OC}}$ from simulation if these values are indeed the minority carrier lifetime. For device simulation, reasonable input parameters currently known for kesterites (discussed in detail in the Supporting Information), with a range of interface recombination velocities in addition to variations in the mobility gap were considered. A reduced mobility gap can be the result of potential fluctuations/band tailing in the absorber, ${ }^{[25,38,39]}$ all of which have been proposed for kesterites. It is found that the measured PL decay times overestimate the minority carrier lifetime for a given device efficiency (shown in Figure 2) and $V_{\text {oc }}$ (see the Supporting Information) for all bounds on the simulation considered. Similar conclusions were observed by Repins et al. following device simulation. ${ }^{[22]}$ Device simulation and experimental results in chalcopyrites and CdTe demonstrate that sub-nanosecond minority carrier lifetimes are sufficient to achieve power conversion efficiencies (PCE) up to $\approx 13 \%$, ${ }^{34,40]}$ representing the performance level currently reported for kesterites. ${ }^{[41]}$

In this work, we analyze intensity-, temperature-, voltagedependent, and spectrally resolved TRPL data measured on CZTSe and CZTSSe absorbers and devices to determine the origin of the PL decay signal. Absorbers from different processing techniques are considered to generalize our observations. First, an analysis methodology is presented to accurately determine the minority carrier decay time from intensitydependent TRPL data of nonideal semiconductors. For the CZTSe and CZTSSe absorbers, we demonstrate that carrier redistribution processes dominate the TRPL signal for early decay times, illustrating the need for good signal-to-noise ratio when analyzing TRPL data for kesterites. We also show that long decay times observed in high-resolution TRPL data of kesterites are related to surface states. Next, we show that TRPL data are not consistent with a recombination limited PL decay model for the kesterite absorbers and devices. Alternatively, a model for minority carrier trapping/detrapping is shown to be consistent with PL decay signal. From this analysis, minority carrier lifetimes of a few hundred picoseconds are estimated for the measured absorbers, which are further supported from 
steady-state PL measurements, electrical characterization, and device simulation. These results illustrate that TRPL decay times measured for kesterites do not represent the minority carrier lifetime in these materials. The analysis methodology, discussion, and simulation results presented herein are generally applicable to TRPL analysis of semiconductors.

\section{Results and Discussion}

One common feature of kesterite absorbers is a low PL efficiency due to significant nonradiative recombination. For the measured CZTSe device, an internal PL efficiency of $<0.01 \%$ is measured from steady-state PL calibrated to absolute photon numbers. Additionally, TRPL data measured on kesterites are typically reported with poor signal-to-noise ratio, with PL decays generally resolved over 1-3 orders of magnitude of signal decay. ${ }^{[5-7,9-12,14,15,18,20-24]}$ As various transport mechanisms can occur within this decay range care must be taken in interpreting such signal.

In contrast, here we report TRPL data, shown in Figure 3, for CZTSe and CZTSSe with up to five orders of magnitude of signal decay, where we find unique features common to both absorbers. A comparison of TRPL data for the CZTSe and CZTSSe absorbers from the distinct processing techniques shows notably similar PL decays for both absorbers. The data can be described by an initial biexponential decay, followed by a long exponential tail of the decay with characteristic decay time of $17 \mathrm{~ns}$. The long tail in the PL decay can be resolved following four orders of magnitude of signal decay and is reported for the first time here. The decay tail is identically observed on absorbers, absorbers passivated with CdS, and devices in highresolution TRPL measurements. Spectrally resolved TRPL data show that the PL emission from the tail region is energetically equivalent to the main PL decay (see the Supporting Information), ruling out this signal originating from a secondary phase or low-energy transition. A comparison of absorbers with

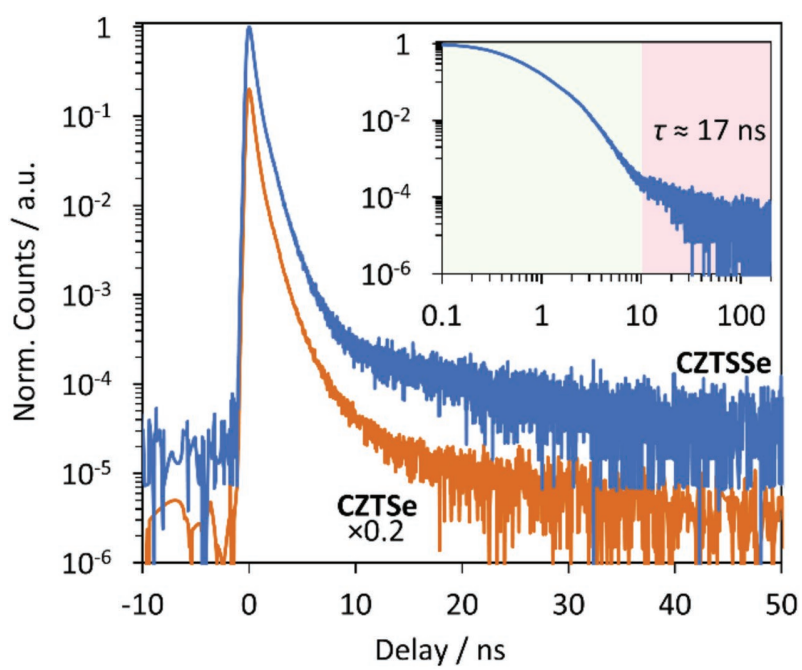

Figure 3. Comparison of TRPL data for CZTSe and CZTSSe $\left(\approx 10^{17} \mathrm{~cm}^{-3}\right.$ carrier injection); CZTSe data are offset for clarity as the decays overlap. CZTSSe data are shown in the inset. different surface treatments to remove oxides shows that this decay tail is related to surface states on the absorber, detailed in the Supporting Information. If this tail is close to the noise level of the signal, an artificially high value for $\tau_{2}$ may be extracted from a biexponential fit to the data.

\subsection{Intensity-Dependent and Spectrally Resolved TRPL}

To describe the measured PL decay, excitation-intensitydependent TRPL was analyzed for both CZTSSe, shown in Figure 4a, and CZTSe (see the Supporting Information); both absorbers yield equivalent results. The excitation intensity was varied from low injection, where the initial carrier generation is lower than the free carrier density $\Delta n_{t=0}<p_{0}$, to high injection where $\Delta n_{t=0}>p_{0}$.

While the bulk of the PL decay signal has a similar characteristic decay time for all injection levels, the initial decay rate for $t<1 \mathrm{~ns}$ increases for increasing injection levels, particularly
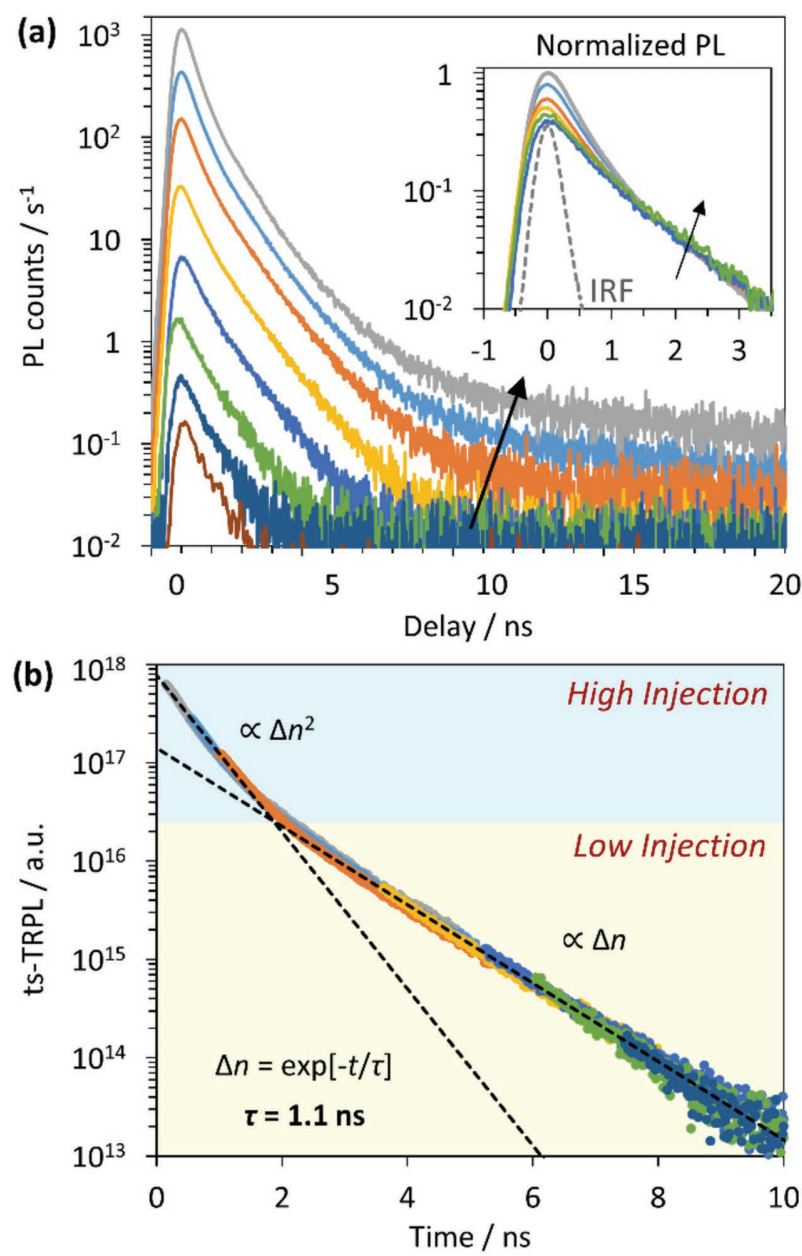

Figure 4. a) Intensity-dependent TRPL data for CZTSSe measured at $\Delta n_{t=0}=\left(1.1 \times 10^{15}, 2.3 \times 10^{15}, 4.5 \times 10^{15}, 9.1 \times 10^{15}, 2.3 \times 10^{16}, 4.5 \times 10^{16}\right.$, $9.1 \times 10^{16}$, and $\left.1.4 \times 10^{17}\right) \mathrm{cm}^{-3}$. Normalized TRPL data are shown in the inset. The arrow indicates increasing injection. The data labeled IRF is the instrument response function. b) Time-shifted TRPL (ts-TRPL) data for CZTSSe computed from data in (a), scaled to the measured $\Delta n_{t=0}$. 
apparent for $\Delta n_{t=0}>6 \times 10^{15} \mathrm{~cm}^{-3}$, shown in the inset of Figure 4. Also, at high injection levels contributions from the long PL decay tail due to surface states can be resolved.

The PL intensity as a function of time $I_{\mathrm{PL}}(t)$ is determined by the radiative emission of carriers ${ }^{[42]} R_{\mathrm{rad}}(t)=B\left[n(t) p(t)-n_{\mathrm{i}}{ }^{2}\right]$, where $B$ is the radiative recombination coefficient, $n(t)=\left(n_{0}+\right.$ $\left.\Delta n_{t=0} \Delta n(t)\right)$ and $p(t)=\left(p_{0}+\Delta p_{t=0} \Delta p(t)\right)$ are the electron and hole densities, respectively, and $n_{\mathrm{i}}{ }^{2}=n_{0} p_{0}$ is the square of the intrinsic carrier density. Accordingly, for symmetric excess carrier generation $\Delta n_{t=0} \Delta n(t)=\Delta p_{t=0} \Delta p(t)$ in a $p$-type semiconductor dominated by non-radiative recombination

$I_{\mathrm{PL}}(t) \propto R_{\mathrm{rad}}(t)=B \Delta n_{t=0}^{2}\left[\Delta n(t)^{2}+\frac{p_{0}}{\Delta n_{t=0}} \Delta n(t)\right]$

In Equation (1), the time-dependence of the PL decay is determined by the rate limiting loss mechanism for minority carriers $\Delta n(t)$. Monomolecular radiative emission dominates at low injection, proportional $\Delta n(t)$, while high-injection conditions are dominated by bimolecular radiative emission at early times, proportional to $\Delta n(t)^{2}$. As the time dependence of the PL intensity is only a function of the excess carrier density, the PL decays taken at various initial carrier injections can be time shifted to match equivalent excess carrier density levels and create a PL decay with improved signal-to-noise ratio. ${ }^{[3]}$ This technique is particularly beneficial for TRPL data which contain long decay tails in the high intensity measurements, such as that measured here for kesterites, where the long decay tail dominates the PL signal for long delay times. The time-shifted TRPL (ts-TRPL) data are shown for CZTSSe in Figure 4b; the long decay tail is not included in the ts-TRPL data for the highintensity measurement data. In contrast to the highest injection measurement in Figure 4a, low values of the excess carrier density $\Delta n(t)$ can now be accurately resolved.

The ts-TRPL data show excellent agreement with Equation (1). At low excess carrier densities, the decay is monomolecular over more than three orders of magnitude where we find exponential behavior of $\Delta n(t)=\exp [-t / \tau]$ with $\tau=1.1 \mathrm{~ns}$ for CZTSSe and $\tau=1.2 \mathrm{~ns}$ for CZTSe. At high excess carrier densities data are in good agreement with a decay rate determined by bimolecular emission $\propto \Delta n(t)^{2}=\exp [-2 t / \tau]$. Accordingly, axial diffusion - which may also affect the PL decay at high injection ${ }^{[2]}$ - should be negligible, indicating a relatively high mobility $\mu_{\mathrm{n}}>75 \mathrm{~cm}^{2} \mathrm{~V}^{-1} \mathrm{~s}^{1}$ for the kesterites (see the Supporting Information). The ts-TRPL data have been uniformly scaled in Figure $4 \mathrm{~b}$ to the measured initial carrier injection $\Delta n_{t=0}$, which allows for determining the free carrier density $p_{0} \approx 1.8 \times 10^{16} \mathrm{~cm}^{-3}$ for CZTSSe. This value is in good agreement with the free carrier density measured by capacitance-voltage profiling on devices measured here (see the Supporting Information) and on equivalently processed CZTSSe devices, ${ }^{[20,43]}$ illustrating the benefit of intensity-dependent TRPL in extracting this parameter for absorbers without the need for device fabrication.

Agreement of the ts-TRPL data with Equation (1) indicates that $\Delta n(t)$ is independent of the carrier injection levels measured. In other words, a single decay time $\tau$ can describe the entire decay of excess carriers. This behavior rules out radiative and Auger recombination limits for $\Delta n(t)$, where $\tau$ varies as a function of $\Delta n \cdot^{[3,44]}$ Additionally, Shockley-Read-Hall (SRH) recombination is expected to be dependent upon the injection level, with the decay time $\tau \approx \tau_{\mathrm{n} 0}$ in low injection and $\tau \approx \tau_{\mathrm{n} 0}+\tau_{\mathrm{p} 0}$ in high injection, ${ }^{[1,26]}$ where $\tau_{\mathrm{n} 0}$ and $\tau_{\mathrm{p} 0}$ are the SRH minority and majority carrier lifetimes, respectively; an intensity-independent decay time would only be expected for $\tau_{\mathrm{p} 0} \ll \tau_{\mathrm{n} 0}$. Intensity-dependent minority carrier lifetimes consistent with SRH recombination have been reported for CIGSe. ${ }^{[45]}$ Alternatively, an exponential decay of $\Delta n(t)$ can be the result of minority carrier detrapping, ${ }^{[27]}$ detailed in Section 2.5.

Closer inspection of the intensity-dependent decay curves yields additional information regarding charge carrier dynamics in the absorber. In Figure $4 \mathrm{~b}$, data for $t<1$ ns in low injection data have not been included in the ts-TRPL data. However, in Figure 5a, the decay curves with the initial decay times are shown. For low injection conditions, a deviation from $\Delta n(t)$ (i.e., Equation (1)) of the measured PL decays for $t<1 \mathrm{~ns}$ can be seen. These deviations are characterized by an increase in the initial decay rate following the excitation pulse. For a given low injection measurement, this initial deviation in PL decay rate is inconsistent with decay data measured at higher initial injection levels, where equivalent $\Delta n(t)$ values are probed following an arbitrary
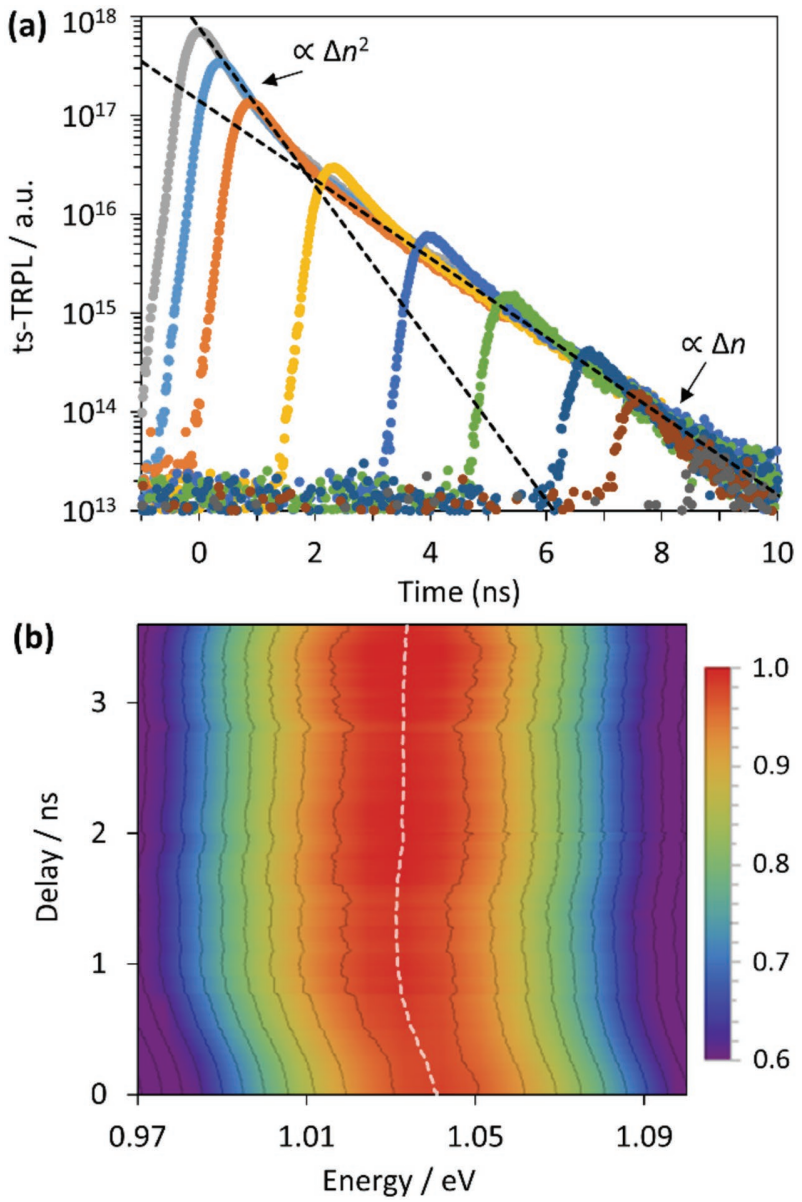

Figure 5. a) Time-shifted TRPL (ts-TRPL) data for CZTSSe computed from data in (a), scaled to the measured $\Delta n_{t=0}$. b) Normalized emissionenergy-dependent TRPL data for CZTSSe; the white dashed line indicates the PL peak position. 
time after excitation (illustrated by the ts-TRPL data). Therefore, we attribute the decay rate for $t<1 \mathrm{~ns}$ to a mechanism which depends on the time delay following excitation, as opposed to the absolute magnitude of $\Delta n(t)$. Surface recombination contributions typically result in an overall decreased PL decay time, $, 2,46]$ though high surface recombination velocities or a low absorber mobility may also lead to a faster initial PL decay. ${ }^{[2]}$ At the same time, surface recombination contributions are expected to be independent of the carrier injection level ${ }^{[2]}$ or be reduced with increasing injection as defect states and/or surface band bending becomes saturated/screened. Since we observe an increasing contribution from the initial decay component with increasing initial injection level, we conclude that surface recombination is not responsible for this increased initial decay rate.

To further investigate early times in the PL decay, emissionenergy-dependent TRPL data were measured for the kesterite absorbers, shown for CZTSSe in Figure 5b. Here, we find that the PL emission shifts to lower energy during the first $1 \mathrm{~ns}$ of the PL decay. This behavior occurs for both absorbers, with a red shift of the PL peak of $\approx 10 \mathrm{meV}$. As similar behavior occurs for samples from distinct processing and varying compositionswith no intentional band gap grading introduced-energetic relaxation of carriers to local minima in band edge fluctuations during initial carrier redistribution, as illustrated in Figure 1a, is attributed to this behavior. Band edge fluctuations in this energy range were reported from PL measurements of kesterites with high Se content. ${ }^{[47]}$ Carrier redistribution is indeed a function of delay time following the excitation pulse rather than $\Delta n(t)$. Additionally, the energetic relaxation of carriers occurs on a similar time scale to the observed initial deviation in PL decay. Therefore, this process is proposed to contribute to the PL signal measured for $t<1 \mathrm{~ns}$ in low injection. In high injection, no deviation of the PL decay at early times is apparent as the faster rate $\Delta n(t)^{2}$ dominates the decay.

Despite the relatively ideal exponential behavior identified for $\Delta n(t)$, we have shown that processes such as carrier redistribution and surface trapping can significantly impact analysis of TRPL data. For instance, single TRPL transients can appear as a complex multiexponential decay. In fact, complex decay curves are ubiquitously reported for TRPL analysis of kesterites. ${ }^{[5-24]}$ Such data can be particularly challenging to interpret as carrier redistribution can dominate the decay signal in data with low signal-to-noise ratio. Additionally, long decay tails unrelated to the relevant minority carrier decay mechanism can obscure extraction of $\Delta n(t)$. Accordingly, we would like to stress that intensity-dependent and spectrally resolved TRPL data may be required to distinguish various contributions in the measured PL decay. While an analysis methodology has been presented to accurately determine $\Delta n(t)$ for absorbers with nonideal behavior, determining the origin of this rate limiting decay mechanism requires further investigation as detailed below.

\subsection{Voltage-Dependent TRPL}

The origin of the PL decay signal was further investigated with voltage-dependent TRPL measured on devices. In the presence of an electric field, photogenerated carriers are initially separated, resulting in a drop in the luminescence signal which follows the charge separation rate $\tau_{\mathrm{cs}}{ }^{-1}$. The separation of charges in an electric field depends on the strength of the electric field $E(V)$, minority carrier mobility $\mu_{\mathrm{n}}$, and competing recombination processes, following Equation (2) in low injection ${ }^{[4,37]}$

$\frac{1}{\tau_{\mathrm{PL}}}=\frac{1}{\tau_{\mathrm{n}}}+\frac{1}{\tau_{\mathrm{cs}}}=\frac{1}{\tau_{\mathrm{n}}}+\frac{q \mu_{\mathrm{n}}}{4 k T} E(V)^{2}$

where $\tau_{\mathrm{PL}}$ is the measured PL decay rate. In high injection, the majority carrier lifetime $\tau_{\mathrm{p}}$ and mobility $\mu_{\mathrm{p}}$ are also relevant, yielding $\tau_{\mathrm{n}}^{-1} \rightarrow \tau_{\mathrm{n}}^{-1}+\tau_{\mathrm{p}}^{-1}$ and $\mu_{\mathrm{n}} \rightarrow \mu_{\mathrm{n}}+\mu_{p}$. To probe charge separation in TRPL measurements, a DC voltage bias $V$ can be applied to a completed device, since $E \propto\left(V_{\mathrm{bi}}-V\right)$ where $V_{\mathrm{bi}}$ is the built-in potential. It is clear that TRPL measurements in the presence of an electric field, particularly on completed devices with a strong p-n junction, should result in a voltage-dependent reduction in the measured decay time relative to an absorber with flat energy bands, as shown for such measurements on chalcopyrites. ${ }^{[4,37]}$

The described charge separation effect on the PL decay is illustrated by voltage-dependent TRPL measured on a CIGSe device, shown in Figure 6a. Here, we see a measured decay time on the device which is significantly shorter than that of the bare absorber. A decrease in the charge separation rate is observed in forward bias, and the PL decay time approaches that of the bare absorber as the voltage bias approaches the flat band voltage (i.e., $E \rightarrow 0$ ), as described by Equation (2). A long tail in the voltage-dependent TRPL measured on the CIGSe device is also observed here due to the high resolution of our measurement system, which is in good agreement with theoretically predicted charge storage effects when measuring TRPL in the presence of a space-charge-region. ${ }^{[2]}$

In contrast to CIGSe, the TRPL data on the investigated kesterites show no dependence on voltage. Voltage-dependent TRPL for the CZTSSe device is shown in Figure 6b, where the decay curves for a voltage bias from -0.8 to $+0.8 \mathrm{~V}$ show identical results. Additionally, PL decays measured on devices are equivalent to that of the bare absorber shown in Figure 3. This behavior illustrates that the presence of an electric field has no impact on the PL decay rate for the kesterite materials studied. A lack of charge separation effects is similarly observed in relatively high-efficiency kesterite devices with $>10 \%$ PCE. ${ }^{[25]}$ It is conceivable that charge separation effects would be negligible for devices with fast recombination rates, where $\tau_{\mathrm{n}}^{-1} \gg q \mu_{\mathrm{n}} E^{2} / 4 k T$ according to Equation (2). However, this condition requires unreasonably low absorber mobilities $\left(\ll 0.08 \mathrm{~cm}^{2} \mathrm{~V}^{-1} \mathrm{~s}^{-1}\right)$ (see the Supporting Information) which do not agree with recent estimates from quantum efficiency $(\mathrm{QE})^{[20]}$ and time-resolved terahertz spectroscopy. ${ }^{[48]}$

The observed lack of charge separation effects reported from TRPL analysis of kesterites demonstrates that the PL decay time for this material cannot be described by a recombination limited processes.

\subsection{Temperature-Dependent TRPL}

To further examine the mechanisms driving the PL decay, temperature-dependent TRPL was measured on the absorbers 

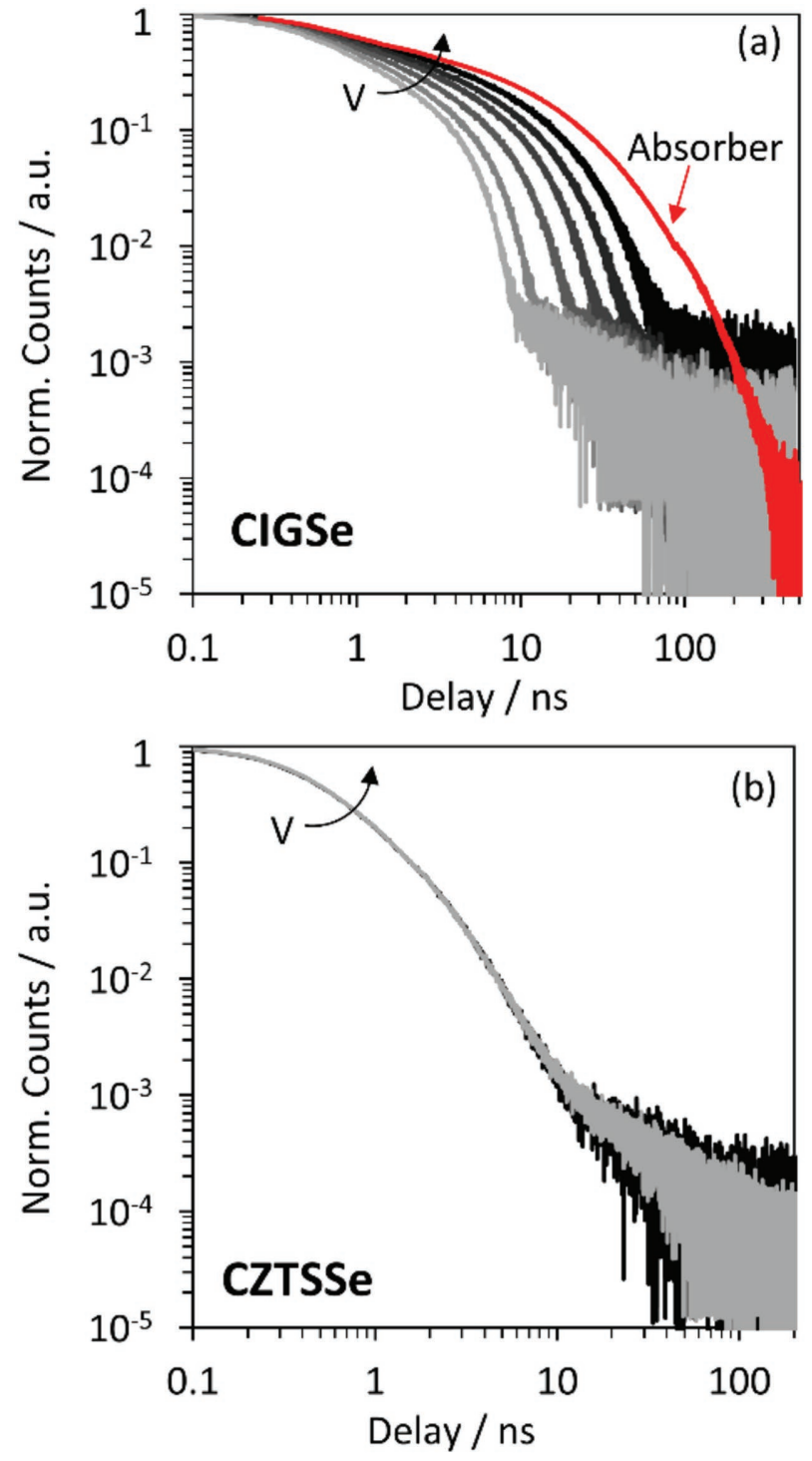

Figure 6. Voltage-dependent TRPL data for a) CICSe and b) CZTSSe devices. The arrow indicates increasing voltage bias on the device, from -0.8 to $+0.5 \vee$ for CICSe and -0.8 to $+0.8 \vee$ for CZTSSe. Equivalent data on the bare absorber are indicated in red for CICSe.

from 90 to $390 \mathrm{~K}$. The minority carrier decay times as a function of temperature are shown in Figure 7 for CIGSe, CZTSe, and CZTSSe. A significantly stronger increase in the decay time is observed with decreasing temperature for the kesterite absorbers in comparison to that measured for CIGSe.

For SRH recombination kinetics, the recombination rate $R_{\mathrm{SRH}}$ of minority carriers in low injection can be described by

$R_{\mathrm{SRH}}=\frac{1}{\tau_{\mathrm{SRH}}(T)}=v_{\mathrm{th}}(T) \sigma_{\mathrm{d}}(T) N$

where $\tau_{\mathrm{SRH}}$ is the SRH recombination lifetime, $v_{\text {th }}$ is the thermal velocity, and $N$ is the density of deep traps with capture cross section $\sigma_{\mathrm{d}}$. In Equation (3), the thermal velocity follows $v_{\text {th }} \propto T^{0.5}$ while the temperature dependence of the $\sigma_{\mathrm{d}}$ is less known. Values of $\sigma_{\mathrm{d}}$ are reported as weakly temperature dependent or

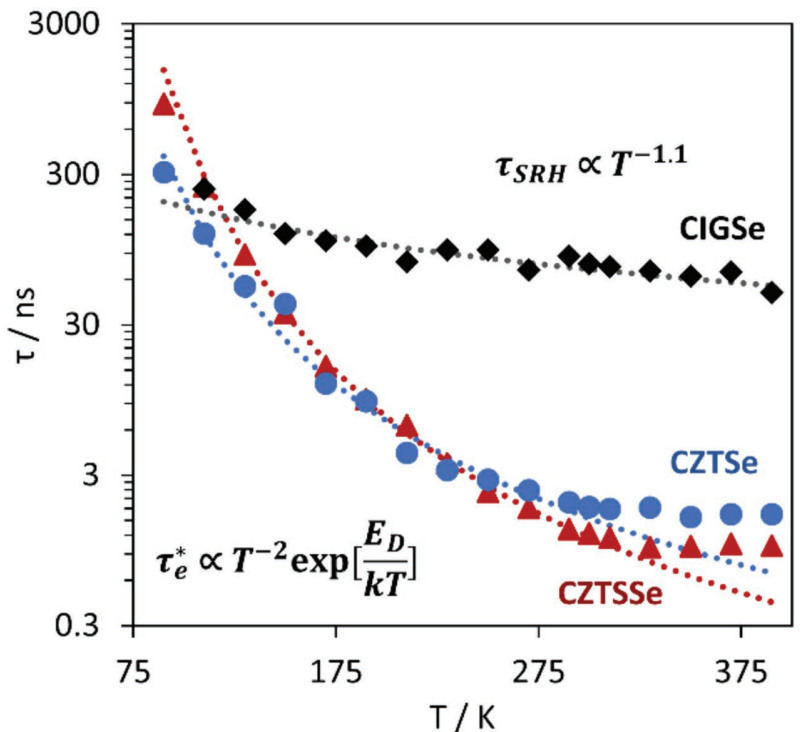

Figure 7. Temperature dependence of the $\mathrm{PL}$ decay time for CIGSe, CZTSe, and CZTSSe. The dashed lines represent a fit to $\tau_{\mathrm{e}}{ }^{*}$ and $\tau_{\mathrm{SRH}}$ for the kesterites and chalcopyrite, respectively.

following a power law dependence $\sigma_{\mathrm{d}} \propto T^{\alpha}$ with $-2.8<\alpha<1$ for various materials. ${ }^{[1,3,32,49-51]}$ A weak temperature dependence of $\sigma_{\mathrm{d}}$ is also commonly found in defect analysis from capacitance spectroscopy. ${ }^{[52-55]}$ For CIGSe, we find $\tau \propto T^{-1.1}$, resulting in $\sigma_{\mathrm{d}} \propto T^{0.6}$, which is in excellent agreement with recently reported temperature-dependent values for $\sigma_{\mathrm{d}}$ in CIGSe. ${ }^{[32]}$ This value is close to $\sigma_{\mathrm{d}} \propto T$ proposed for a circular-shaped localized defect performing harmonic oscillations. ${ }^{[27]}$ In contrast, the kesterite absorbers demonstrate a significantly larger temperature dependence with $\tau \propto T^{-5.4 \pm 0.7}$, resulting in $\sigma_{\mathrm{d}} \propto T^{4.8 \pm 0.7}$ which is outside the range of reported power law dependencies for $\sigma_{\mathrm{d}}$.

Alternatively, the temperature dependence of the PL decay time can exhibit thermally activated behavior with an Arrhenius temperature dependence. In one case, the capture cross section follows $\sigma_{\mathrm{d}} \propto \sigma_{\infty} \exp \left[-E_{\infty} / k T\right]$ for nonradiative capture and emission by multiphonon emission. ${ }^{[56]}$ Such a process would present a dramatic contrast to the nonradiative recombination mechanism observed in CIGSe, and is also contradicted by the deviation from exponential behavior (saturation) observed at high temperatures. Furthermore, a strong temperature dependence of the minority carrier lifetime is not supported by electrical analysis of kesterite devices. Specifically, analysis of the saturation current $J_{0}=J_{00} \times \exp \left[-E_{\mathrm{A}} / k T\right]$ and $V_{\mathrm{OC}}$ modeling indicate a weakly temperature-dependent saturation current prefactor $J_{00}$ for $100 \mathrm{~K} \leq T \leq 300 \mathrm{~K}$, where $J_{00} \propto \tau^{-1}$ or $J_{00} \propto \tau^{-1 / 2}$ for recombination limited in the SCR or neutral bulk absorber, respectively. ${ }^{[57]}$ On the other hand, an exponential dependence on temperature is exactly what is expected from thermal emission of carriers from a trap state, as detailed in Section 2.5.

\subsection{Evidence for a Sub-Nanosecond Minority Carrier Lifetime}

A minority carrier lifetime of a few hundred picoseconds is supported by device simulation (see Figure 2 and the Supporting 
Information), even in the limit of high interface recombination velocities and mobility gap narrowing due to potential fluctuations/band tailing, as device efficiencies and open-circuit voltages in the range reported for kesterites are simulated for $\tau_{\mathrm{n}} \leq 500 \mathrm{ps}$.

The calibrated steady-state PL yield can also be used to estimate the minority carrier lifetime. The value of $\tau_{\mathrm{n}}$ is estimated from the internal PL efficiency $\eta_{\mathrm{PL}, \text { Int }}$, following ${ }^{[42]}$

$\eta_{\mathrm{PL}, \text { Int }}=\frac{\tau_{\mathrm{n}}}{\tau_{\text {Rad }}} \approx \tau_{\mathrm{n}} B p_{0}$

where $\tau_{\text {Rad }}$ is the radiative lifetime. For CZTSe, the steadystate PL yield, calibrated to absolute photon numbers, was measured under $660 \mathrm{~nm}$ excitation with AM1.5 illumination intensity. Accounting for the emission cone of the measured external luminescence, the internal PL yield was calculated at $\eta_{\mathrm{PL}, \mathrm{Int}} \approx 7.2 \times 10^{-5}$. Additionally, using reasonable input parameters for CZTSe, we find $B \approx 7.4 \times 10^{-12}$ to $1.7 \times 10^{-10} \mathrm{~cm}^{-3} \mathrm{~s}^{-1}$, which results in $\tau_{\mathrm{n}} \approx 280 \pm 250 \mathrm{ps}$ for CZTSe photoluminescence measured at $305 \pm 5 \mathrm{~K}$. Detailed calculations are provided in the Supporting Information. In other words, the low PL yield observed in kesterite absorbers is a result of a low minority carrier lifetime in this material.

Finally, using recent estimates of the minority carrier mobility $\mu_{\mathrm{n}} \approx 100 \mathrm{~cm}^{2} \mathrm{~V}^{-1} \mathrm{~s}^{-1},{ }^{[48]}$ reference saturation current values from bulk recombination $J_{00} \approx 6.4 \times 10^{5}$ to $3.2 \times 10^{7} \mathrm{~mA} \mathrm{~cm}^{-2}$ are estimated for the $\tau_{\mathrm{n}}$ values reported here (see the Supporting Information), where $J_{00}$ is an important parameter causing the high saturation current and thus low $V_{\mathrm{OC}}$ commonly reported for kesterite devices. This estimate of $J_{00}$ is in good agreement with values reported from recent temperature-dependent current-voltage (JVT) measurements on CZTSSe devices similar to that measured here $\left(J_{00} \approx 10^{6} \mathrm{~mA} \mathrm{~cm}^{-2}\right) \cdot{ }^{[57]}$

These results illustrate that the minority carrier lifetime is expected to be below that of the measured PL decay time for the kesterite absorbers. This analysis is particularly relevant for kesterite absorbers where minority carrier lifetimes in the nanosecond regime-with values as high as 10-20 ns-are reported from the PL decay time, as the PL yield should be consistent with the minority carrier lifetime.

\subsection{Minority Carrier Trapping/Detrapping}

To reconcile the measured TRPL behavior and evidence for $\tau_{\mathrm{n}}$ below the measured PL decay time with a consistent decay mechanism for $\Delta n(t)$, we propose that minority carrier trapping/detrapping is responsible for the PL decay in the kesterite absorbers. Following the formulations by Maiberg et al. ${ }^{[27]}$ and Ahrenkiel et al. ${ }^{[28]}$ for charge carrier dynamics in the presence of minority carrier trapping, photogenerated minority carriers are initially trapped into localized shallow state(s) near the conduction band edge, with capture times smaller or close to that of the minority carrier recombination lifetime $\left(\tau_{\mathrm{c}} \leq \tau_{\mathrm{n}}\right)$. Subsequently, the trapped minority carriers are slowly released back to the conduction band with the emission time $\tau_{\mathrm{e}}$. For trap emission times longer than the minority carrier lifetime, a minority carrier trapping mechanism results in an initially fast decay time governed by $\tau_{\mathrm{c}}$, followed by a longer decay time $\tau_{e}{ }^{*}$ governed by the trap emission time $\tau_{e}{ }^{*}=\tau_{\mathrm{e}} \times\left(\tau_{\mathrm{n}} / \tau_{\mathrm{c}}\right) \cdot{ }^{[27]} \mathrm{It}$ should be noted that the trap emission dominated decay time $\tau_{e}^{*}$ is scaled by the minority carrier lifetime, therefore relative changes in $\tau_{\mathrm{n}}$ may be reflected in the TRPL data, though the magnitude of this value does not represent the actual value of $\tau_{\mathrm{n}}$ or $\tau_{\mathrm{e}}$.

While minority carrier detrapping from surface states is observed for the long PL decay tail with $\tau_{e}^{*} \approx 17 \mathrm{~ns}$, minority carrier detrapping can also dominate the decay on shorter time scales, such as $\tau_{e}^{*} \approx 1.1 \mathrm{~ns}$, for materials with a low minority carrier lifetime. Therefore, the evidence for a sub-nanosecond minority carrier lifetime, smaller than the measured PL decays times for kesterites, is consistent with a minority carrier detrapping process limiting the decay of excess carriers. Additionally, the drift of carriers in an electric field has no influence on carriers localized in a trap state. This behavior is consistent with the voltage-dependent TRPL results where charge separation is shown to have no influence on the measured PL decay. Finally, minority carrier detrapping results in an exponential decay of $\Delta n(t)$, where the decay time $\tau_{e}^{*}$ is expected to remain constant for injection levels below the total trap density. This behavior is fully consistent with the measured intensity-dependent TRPL behavior, where a single decay time is observed for $\Delta n(t)^{2}$ in high injection and $\Delta n(t)$ in low injection. Yet for the measured absorbers, a clear drop in the initial PL decay signal due to rapid trap capture is not observed. However, as carrier generation occurs for $\pm 3 \tau_{\mathrm{p}} \approx \pm 100 \mathrm{ps}$ around the excitation pulse peak, and the instrument response function is limited to $\approx 250 \mathrm{ps}$, rapid trap capture may not be resolved in the TRPL measurements. Additionally, trap capture processes may also contribute to the initial PL decays ( $t<1 \mathrm{~ns}$ ) which were shown to deviate from $\Delta n(t)$ concurrent with the energetic relaxation of carriers. We note that similar minority carrier trapping/detrapping mechanisms with emission times on the nanosecond time scale may also be prevalent for the CIGSe material system, however due to the long minority carrier lifetime of CIGSe relative to kesterites (with $\tau_{\mathrm{n}}$ in the $10-100$ ns range), ${ }^{[32]}$ such trapping effects will not dominate the TRPL measurement.

The temperature dependence of $\tau_{e}^{*}$ is determined by the thermal emission of carriers from the trap state, following ${ }^{[27,58]}$

$e_{\mathrm{n}}^{*}=\frac{1}{\tau_{e}^{*}(T)}=v_{\mathrm{th}}(T) \sigma_{\mathrm{d}}(T) N_{\mathrm{c}}(T) \exp \left[-E_{\mathrm{D}} / k T\right]$

where $\tau_{e}^{*}$ is the effective electron emission rate, ${ }^{[59]} N_{\mathrm{C}} \propto T^{1.5}$ is the conduction band effective density of states and $E_{\mathrm{D}}$ is the energetic distance between the conduction band and trap level. It can be seen in Figure 7 that the kesterite absorbers are consistent with the behavior for trap emission dominated decay times $\left(\tau_{\mathrm{e}} \propto T^{-2} \exp \left[E_{\mathrm{D}} / k T\right]\right)$ in the range $90<T<330 \mathrm{~K}$. Assuming negligible temperature dependence of $\sigma_{\mathrm{d}}$ for SRH recombination, ${ }^{[59]}$ we find that CZTSSe and CZTSe are thermally activated by $E_{\mathrm{D}}=53 \pm 1 \mathrm{meV}$ and $E_{\mathrm{D}}=35 \pm 3 \mathrm{meV}$, respectively.

At high temperature ( $T \geq 330 \mathrm{~K}$ ), a qualitative change in the measured PL decay is observed for the extracted decay times. This behavior further supports the proposed minority carrier detrapping mechanism, as the PL decay time should approach 
the minority carrier lifetime at elevated temperature due to the increased emission rate of carriers from the trap state(s). However, emission times of a few hundred picoseconds are still expected at $390 \mathrm{~K}$ in our analysis, indicated by the dashed lines in Figure 7. Accordingly, the competing processes such as recombination, trap capture, and carrier redistribution cannot be unambiguously distinguished even at our highest temperature measured. Following Maiberg et al., ${ }^{[27]}$ emission, capture, and recombination processes which occur on a similar time scale will result in a measured PL decay time longer than the respective individual contributions.

The intensity-, voltage-, temperature-dependent, and spectrally resolved results discussed herein show that if minority carrier trapping is ignored, the minority carrier lifetime can be severely overestimated from TRPL analysis. In light of device simulation results and the published PL decay behavior for kesterite absorbers and devices, we believe this is a common result in TRPL analysis reported for kesterites. These results further support ${ }^{[20,25,39,57]}$ that the low performance in kesterites can be explained by the bulk properties of the absorber. While bandtailing/potential fluctuations have been shown to contribute to $V_{\mathrm{OC}}$ limitations in kesterite devices, ${ }^{[20,25,57]}$ here we demonstrate that a low minority carrier lifetime is also responsible for significant performance limitations in these materials.

\section{Conclusion}

In conclusion, we have demonstrated how identifying the minority carrier lifetime in nonideal semiconductors can be challenging due to complex charge carrier dynamics. For kesterites, minority carrier trapping, surface effects, and energetic relaxation of carriers are shown to severely affect the PL transient. Therefore, characteristic PL decay times extracted from TRPL measurements at room temperature are often unrelated to the minority carrier lifetime for these materials. Here, we estimate minority carrier lifetimes at $300 \mathrm{~K}$ in the measured CZTSe and CZTSSe absorbers from distinct processing techniques to be on the order of hundreds of picoseconds, rather than the few nanoseconds corresponding to PL decay times measured here and reported elsewhere for kesterites. This conclusion is supported from detailed TRPL analysis, steady-state PL efficiency measurements, electrical characterization, comparison with recombination-limited CIGSe, as well as device simulation.

An analysis methodology is presented to determine the decay rate of excess minority carriers and to investigate the origin of this rate limiting decay mechanism for materials with nonideal behavior. First, time-shifted analysis of intensitydependent TRPL can be used to isolate various contributions to the PL decay and accurately determine the excess carrier decay rate and free carrier density on absorbers. To determine if this excess carrier decay is limited by recombination:

- Intensity-dependent TRPL should be consistent with the expected recombination lifetime in both low and high injection.

- TRPL measured on devices should be consistent with chargeseparation effects expected for the carrier mobility.
- Temperature-dependent TRPL should be consistent with reasonable carrier capture properties in defects and the recombination behavior measured from electrical characterization (e.g., $\left.J_{0}(T)\right)$.

- The steady-state PL yield should be consistent with minority carrier lifetime.

Through this analysis of kesterite absorbers from distinct processing techniques, and comparison with TRPL data from literature, we assert that minority carrier trapping is responsible for the PL decay times generally reported for kesterites. Additional analysis with consistent results should be checked individually when long lifetimes are reported for this material. We conclude that a sub-nanosecond minority carrier lifetime in kesterite materials is the present limitation to device performance.

\section{Experimental Section}

CZTSe absorbers and devices were fabricated from the selenization of stacked metallic precursors (see the Supporting Information). CZTSSe absorbers and devices were fabricated from the selenization of nanocrystal-based precursors, as described by Miskin et al. ${ }^{[60]}$ with a $[S] /([S]+[S e])$ ratio of 5-10 at\%. CZTSe and CZTSSe devices from these processes exhibit PCE as high as $9 \%$. Absorber formation, buffer layer deposition, and device completion were performed independently on the CZTSe and CZTSSe absorbers at HZB and Purdue, respectively. Devices with $\approx 8 \%$ PCE, without antireflective coating, were measured herein; additional device information can be found in the Supporting Information. For comparison, a CICSe device/absorber ( $16 \%$ PCE) was measured, which was fabricated from multistage coevaporation. ${ }^{[6]]}$

Device simulation was performed in SCAPS 3.2.01.[62] TRPL measurements were performed in a custom setup which uses a $660 \mathrm{~nm}$ wavelength pulsed laser source and time correlated single photon counting with an InGaAs photomultiplier calibrated from 900 to $1440 \mathrm{~nm}$ with an instrument response function less than $250 \mathrm{ps}$. $\mathrm{PL}$ emission from the samples is either taken panchromatically with a long-pass filter for the laser line or monochromatically with a full width at half maximum (FWHM) resolution $<2 \mathrm{~nm}$. Signal excitation and collection were achieved with a beam splitter/reflective objective combination, which eliminates chromatic aberration when exciting and collecting the excitation and emission signals and is optimized for maximum emission energy signal. An excitation spot with Gaussian profile of diameter $23 \mu \mathrm{m}$ was used, with laser pulse frequencies between $500 \mathrm{kHz}$ and $2.5 \mathrm{MHz}$; the incident photon flux has Gaussian time dependence with pulse length ${ }^{[2]} \tau_{\mathrm{p}} \approx 34 \mathrm{ps}$. Carrier injection density per pulse (reported throughout) was calculated using absorption data from voltage-dependent $\mathrm{QE}$ measurements on the relevant devices, according to Hages et al. ${ }^{[20]}$ Temperature-dependent measurements were performed in an evacuated closed cycle He cryostat, all other measurements were performed in air under ambient conditions unless specifically noted. TRPL data were background subtracted. Following device measurements, the window/buffer layers were removed with a $1 \min 1 \mathrm{M} \mathrm{HCl}$ etch for measurements on the bare absorber. The $\mathrm{HCl}$ etch was performed prior to all measurements on absorbers to remove effects of long-term degradation due to surface oxidation; measurements on fresh absorbers show identical properties. Intensity-, voltage-dependent, and spectrally resolved TRPL data are shown in the main body of this work for CZTSSe, though equivalent data for CZTSe can be found in the Supporting Information. Calibrated steadystate PL measurements were performed in the system described by Redinger et al. ${ }^{[63]}$ 


\section{Supporting Information}

Supporting Information is available from the Wiley Online Library or from the author.

\section{Acknowledgements}

The authors would like acknowledge Horizon STARCELL2020 (NMBP-03 2016-720907) for financial support. R.A. thanks SERIIUSDE-08G028308 for financial support. A.R. thanks the Fonds National de la Recherche Project No. 7842175 for financial support. The authors also would like to thank Steffen Kretzschmar for fruitful discussion.

\section{Conflict of Interest}

The authors declare no conflict of interest.

\section{Keywords}

kesterites, minority carrier lifetime, solar cells, time-resolved photoluminescence, trapping

Received: January 17, 2017 Revised: March 14, 2017 Published online:

[1] R. K. Ahrenkiel, in Minority Carriers in III-V Semiconductors: Physics and Applications. (Eds: R. K. Ahrenkiel and M. S. Lundstrom), Academic, San Diego, CA 1993, pp. 39-150.

[2] M. Maiberg, R. Scheer, J. Appl. Phys. 2014, 116, 123711.

[3] B. C. Connelly, G. D. Metcalfe, H. Shen, M. Wraback, Appl. Phys. Lett. 2010, 97, 251117.

[4] D. Kuciauskas, J. V. Li, A. Kanevce, H. Guthrey, M. Contreras, J. Pankow, P. Dippo, K. Ramanathan, J. Appl. Phys. 2015, 117, 185102.

[5] S. G. Haass, M. Diethelm, M. Werner, B. Bissig, Y. E. Romanyuk, A. N. Tiwari, Adv. Energy Mater. 2015, 5, 1500712.

[6] Y. S. Lee, T. Gershon, O. Gunawan, T. K. Todorov, T. Gokmen, Y. Virgus, S. Guha, Adv. Energy Mater. 2015, 5, 1401372.

[7] G. Brammertz, M. Buffière, S. Oueslati, H. ElAnzeery, K. B. Messaoud, S. Sahayaraj, C. Köble, M. Meuris, J. Poortmans, Appl. Phys. Lett. 2013, 103, 163904.

[8] J. Li, H. Wang, L. Wu, C. Chen, Z. Zhou, F. Liu, Y. Sun, J. Han, Y. Zhang, ACS Appl. Mater. Interfaces 2016, 8, 10283.

[9] O. Gunawan, T. K. Todorov, D. B. Mitzi, Appl. Phys. Lett. 2010, 97, 233506

[10] S. Bag, O. Gunawan, T. Gokmen, Y. Zhu, D. B. Mitzi, Chem. Mater 2012, 24, 4588

[11] S. Bag, O. Gunawan, T. Gokmen, Y. Zhu, T. K. Todorov, D. B. Mitzi, Energy Environ. Sci. 2012, 5, 7060.

[12] K. B. Messaoud, M. Buffière, G. Brammertz, H. ElAnzeery, S. Oueslati, J. Hamon, B. J. Kniknie, M. Meuris, M. Amlouk, J. Poortmans, Prog. Photovoltaics Res. Appl. 2015, 23, 1608.

[13] C. J. Hages, M. J. Koeper, R. Agrawal, Sol. Energy Mater. Sol. Cells 2016, 145, 342

[14] I. Repins, C. Beall, N. Vora, C. DeHart, D. Kuciauskas, P. Dippo, B. To, J. Mann, W.-C. Hsu, A. Goodrich, R. Noufi, Sol. Energy Mater. Sol. Cells 2012, 101, 154

[15] J. V. Li, D. Kuciauskas, M. R. Young, I. L. Repins, Appl. Phys. Lett. 2013, 102, 163905.
[16] T. Gokmen, O. Gunawan, D. B. Mitzi, J. Appl. Phys. 2013, 114, 114511.

[17] T. K. Todorov, J. Tang, S. Bag, O. Gunawan, T. Gokmen, Y. Zhu, D. B. Mitzi, Adv. Energy Mater. 2013, 3, 34.

[18] M. Buffiere, G. Brammertz, A. El Mel, N. Lenaers, Y. Ren, A. E. Zaghi, Y. Mols, C. Koeble, J. Vleugels, M. Meuris, J. Poortmans, in 2013 IEEE 39th Photovoltaic Specialists Conf., IEEE, 2013, Tampa, FL, USA, pp. 1941-1944.

[19] C. J. Hages, S. Levcenco, C. K. Miskin, J. H. Alsmeier, D. Abou-Ras, R. G. Wilks, M. Bär, T. Unold, R. Agrawal, Prog. Photovoltaics Res. Appl. 2015, 23, 376

[20] C. J. Hages, N. J. Carter, R. Agrawal, J. Appl. Phys. 2016, 119, 14505.

[21] C. J. Hages, M. J. Koeper, C. K. Miskin, K. W. Brew, R. Agrawal, Chem. Mater. 2016, 28, 7703.

[22] I. L. Repins, H. Moutinho, S. G. Choi, A. Kanevce, D. Kuciauskas, P. Dippo, C. L. Beall, J. Carapella, C. DeHart, B. Huang, S. H. Wei, J. Appl. Phys. 2013, 114, 84507

[23] H. Cui, C. Lee, W. Li, X. Liu, X. Wen, X. Hao, Int. J. Photoenergy 2015, 2015, 1

[24] D. A. R. Barkhouse, O. Gunawan, T. Gokmen, T. K. Todorov, D. B. Mitzi, Prog. Photovoltaics Res. Appl. 2012, 20, 6.

[25] T. Gokmen, O. Gunawan, T. K. Todorov, D. B. Mitzi, Appl. Phys. Lett. 2013, 103, 103506

[26] M. Maiberg, R. Scheer, J. Appl. Phys. 2014, 116, 123710.

[27] M. Maiberg, T. Hölscher, S. Zahedi-Azad, R. Scheer, J. Appl. Phys. 2015, 118, 105701

[28] R. K. Ahrenkiel, N. Call, S. W. Johnston, W. K. Metzger, Sol. Energy Mater. Sol. Cells 2010, 94, 2197.

[29] A. Kanevce, D. H. Levi, D. Kuciauskas, Prog. Photovoltaics Res. Appl. 2014, 22, 1138

[30] R. K. Ahrenkiel, J. Appl. Phys. 1987, 62, 2937.

[31] W. K. Metzger, R. K. Ahrenkiel, J. Dashdorj, D. J. Friedman, Phys. Rev. B 2005, 71, 35301.

[32] A. Redinger, S. Levcenko, C. J. Hages, D. Greiner, C. A. Kaufmann, T. Unold, Appl. Phys. Lett. 2017, 110, 122104

[33] I. L. Repins, B. Egaas, L. M. Mansfield, M. A. Contreras, C. P. Muzzillo, C. Beall, S. Glynn, J. Carapella, D. Kuciauskas, Rev. Sci. Instrum. 2015, 86, 13907.

[34] I. Repins, L. Mansfield, A. Kanevce, S. A. Jensen, D. Kuciauskas, S. Glynn, T. Barnes, W. Metzger, J. Burst, C. Jiang, P. Dippo, S. Harvey, G. Teeter, C. Perkins, B. Egaas, A. Zakutayev, J. Alsmeier, T. Lusky, L. Korte, R. G. Wilks, M. Bar, Y. Yan, S. Lany, P. Zawadzki, J. Park, S. Wei, in 2016 IEEE 43rd Photovoltaic Specialists Conf., IEEE, 2016, Portland, OR, USA, pp. 0309-0314.

[35] I. L. Repins, W. K. Metzger, C. L. Perkins, J. V. Li, M. A. Contreras, IEEE Trans. Electron Devices 2010, 57, 2957.

[36] W. K. Metzger, I. Repins, M. Romero, P. Dippo, M. A. Contreras, R. Noufi, D. Levi, Thin Solid Films 2009, 517, 2360.

[37] M. Maiberg, C. Spindler, E. Jarzembowski, R. Scheer, Thin Solid Films 2015, 582, 379

[38] T. Tiedje, Appl. Phys. Lett. 1982, 40, 627

[39] J. E. Moore, C. J. Hages, R. Agrawal, M. S. Lundstrom, J. L. Gray, Appl. Phys. Lett. 2016, 109, 21102.

[40] R. Scheer, H.-W. Schock, Chalcogenide Photovoltaics: Physics, Technologies, and Thin Film Devices, Wiley-VCH, Weinheim, Germany 2011.

[41] W. Wang, M. T. Winkler, O. Gunawan, T. Gokmen, T. K. Todorov Y. Zhu, D. B. Mitzi, Adv. Energy Mater. 2014, 4, 1301465.

[42] T. Unold, L. Gütay, in Advanced Characterization Techniques for Thin Film Solar Cells (Eds: D. Abou-Ras, T. Kirchartz, and U. Rau, 1st), Wiley VCH, Weinheim, Germany 2011, pp. 151-175.

[43] C. J. Hages, N. J. Carter, J. Moore, S. M. McLeod, C. K. Miskin, M. S. Lundstrom, R. Agrawal, C. Joglekar, M. S. Lundstrom, R. Agrawal, in Proc. 39th IEEE Photovoltaics Specialists Conf., IEEE, 2013, Tampa, FL, USA, p. 1966. 
[44] F. Staub, H. Hempel, J.-C. Hebig, J. Mock, U. W. Paetzold, U. Rau, T. Unold, T. Kirchartz, Phys. Rev. Appl. 2016, 6, 44017.

[45] S. Puttnins, S. Levcenco, K. Schwarzburg, G. Benndorf, F. Daume, A. Rahm, A. Braun, M. Grundmann, T. Unold, Sol. Energy Mater. Sol. Cells 2013, 119, 281

[46] R. K. Ahrenkiel, Solid State Electron. 1992, 35, 239.

[47] S. Levcenko, J. Just, A. Redinger, G. Larramona, S. Bourdais, G. Dennler, A. Jacob, T. Unold, Phys. Rev. Appl. 2016, 5, 24004.

[48] H. Hempel, A. Redinger, I. Repins, C. Moisan, G. Larramona, G. Dennler, M. Handwerg, S. F. Fischer, R. Eichberger, T. Unold, J. Appl. Phys. 2016, 120, 175302.

[49] S. Levcenko, V. E. Tezlevan, E. Arushanov, S. Schorr, T. Unold, Phys. Rev. B 2012, 86, 45206.

[50] G. Ascarelli, S. Rodriguez, Phys. Rev. 1961, 124, 1321.

[51] M. S. Tyagi, R. Van Overstraeten, Solid State Electron. 1983, 26, 577.

[52] J. R. Heath, P. Zabierowski, in Advanced Characterization Techniques for Thin Film Solar Cells (Eds: D. Abou-Ras, T. Kirchartz, and U. Rau, 1st), Wiley VCH, Weinheim, Germany 2011, pp. 81-105.

[53] T. Walter, R. Herberholz, C. Müller, H. W. Schock, J. Appl. Phys. 1996, 80, 4411.
[54] O. Gunawan, T. Gokmen, C. W. Warren, J. D. Cohen, T. K. Todorov, D. A. R. Barkhouse, S. Bag, J. Tang, B. Shin, D. B. Mitzi, Appl. Phys. Lett. 2012, 100, 253905.

[55] J. T. Heath, J. D. Cohen, W. N. Shafarman, J. Appl. Phys. 2004, 95, 1000.

[56] C. H. Henry, D. V. Lang, Phys. Rev. B 1977, 15, 989.

[57] C. J. Hages, N. J. Carter, R. Agrawal, T. Unold, J. Appl. Phys. 2014, 115, 234504.

[58] D. V. Lang, J. Appl. Phys. 1974, 45, 3023.

[59] Due to the relation $\tau_{e}^{*}=\tau_{\mathrm{e}} \times\left(\tau_{\mathrm{n}} / \tau_{\mathrm{c}}\right)$, the effective electron emission rate $e_{n}{ }_{n}$ is a function of $\sigma_{\mathrm{d}}$ according to $\tau_{n}$, as opposed to the capture cross section of the trap state (see the Supporting Information).

[60] C. K. Miskin, W.-C. Yang, C. J. Hages, N. J. Carter, C. S. Joglekar, E. A. Stach, R. Agrawal, Prog. Photovoltaics Res. Appl. 2015, 23, 654.

[61] D. Greiner, J. Lauche, S. Harndt, R. Klenk, R. Schlatmann, C. A. Kaufmann, in 2015 IEEE 42nd Photovoltaic Specialists Conf., IEEE, New Orleans, LA, USA 2015, pp. 1-6.

[62] M. Burgelman, K. Decock, S. Khelifi, A. Abass, Thin Solid Films 2013, 535, 296.

[63] A. Redinger, S. Kretzschmar, T. Unold, in 2016 IEEE 43rd Photovoltaic Specialists Conf., IEEE, Portland, OR, USA 2016, pp. 3559-3562. 